Proc. Indian Acad. Sci. (Chem. Sci.), Vol. 105, No. 6, December 1993, pp. 527-538.

(C) Printed in India.

\title{
Photoinduced electron transfer reactions of trans-stilbene surfactants in Langmuir-Blodgett assemblies and phospholipid bilayers
}

\author{
DAVID G WHITTEN*, INNA FURMAN, CRISTINA GEIGER, \\ WANDA RICHARD and SUSAN P SPOONER \\ Department of Chemistry, University of Rochester, Rochester, New York 14627, USA
}

\begin{abstract}
The photochemistry and photophysics of a series of stilbene-functionalized fatty acids (SFA) have been examined in homogeneous solutions, supported Langmuir-Blodgett assemblies, and as guests in phospholipid bilayers and micellar dispersions. The spectroscopic characteristics of stilbene-functionalized derivatives of phosphatidyl choline (SFA-PC) have also been examined in organic solvents, aqueous dispersions and aqueous solution containing an excess of dipalmitoyl choline (DPPC), as well as in aqueous-methanol solutions containing $\gamma$-cyclodextrin. Langmuir-Blodgett assemblies of individual SFAs, as well as mixtures of various SFAs, exhibit spectroscopic properties (blue-shifted absorbance and red-shifted fluorescence relative to those observed in organic solvents) consistent with the formation of an " $\mathrm{H}$ " aggregate. The same effect is observed for SFA-PCs in aqueous dispersions and in aqueous-methanol solutions containing $\gamma$-cyclodextrin. The " $\mathrm{H}$ " aggregate is found to be the preferred geometric orientation of the stilbene chromophores which may correspond to an energy minimum for the systems investigated. Preliminary studies with SFA-PC in methanol-water solutions containing $\gamma$-cyclodextrin suggest that an association of only two stilbene chromophores is required to form the " $\mathrm{H}$ " aggregate.
\end{abstract}

Keywords. Photoinduced electron transfer; stilbene surfactants; Langmuir-Blodgett assemblies; phospholipid bilayers.

\section{Introduction}

The stilbene chromophore is one of the most widely investigated conjugated organic systems, due both to its characteristic and rich photochemistry and its spectroscopic accessibility (Hammond et al 1964; Saltiel et al 1975; Saltiel and Charlton 1980; Itoh and Kohler 1987; Allen and Whitten 1989; Saltiel 1992). In recent years, we and others have focused considerable attention towards the photophysics and photochemistry of substituted stilbenes and related molecules incorporated as guests in a variety of microheterogeneous media (Geiger and Turro 1977; Russell et al 1980, 1981; Brown et al 1985; Suddaby et al 1985). The sensitivity of the stilbene chromophore towards viscosity and local order results in its serving as a fairly sensitive probe to the local environment provided by these media; dramatic effects have been observed on both the thermal and photoinduced reactions of the stilbene chromophore in these media in a number of different investigations (Brown et al 1985; Mizutani and Whitten 1985; Takagi et al 1986). Incorporation of the trans-stilbene chromophore into a saturated fatty acid results in an amphiphile which is expected to form assemblies "anchoring"

* For correspondence 
the stilbene chromophore in a relatively hydrophobic and ordered site, especially in Langmuir-Blodgett and various bilayer assemblies in aqueous media. In fact, we have found that the "stilbene fatty acids" (SFAs) are excellent surfactants which have film-forming properties very similar to the corresponding saturated fatty acids having the same overall lengths (Mooney 1983; Mooney et al 1984). In examining the compression, for example, of SFA films at the air-water interface, the behavior and limiting dimensions are found to be almost indistinguishable from corresponding saturated fatty acids when either pure SFA or SFA-fatty acid mixtures are examined. However, our earliest investigations of these films revealed that both in compressed films at the air-water interface and in assemblies supported on rigid optically transparent supports (formed via sequential transfer of films from the air-water interface) the stilbene chromophore shows evidence of aggregation which sharply modifies the absorption and photophysics of the parent chromophore (Mooney 1983; Mooney et al 1984; Mooney and Whitten 1986). In the present manuscript, we discuss the behavior of these SFAs in several media with emphasis on their reactivities in transferred supported multilayers.

\section{Results and discussion}

\subsection{General properties of the SFAs}

Chart 1 illustrates the structures of several of the SFAs used in this study and shows the codes used in representing them. These stilbene derivatives can all be incorporated into fluid solutions of microheterogeneous media such as detergent micelles or phospholipid bilayers. Incorporation into sodium dodecylsulfate micelles results in reactivity very similar to that observed in homogeneous organic solvents. Absorption and fluorescence spectra characteristic of the stilbene monomer are observed and the fluorescence and isomerization efficiencies are comparable to those observed in nonviscous homogeneous solutions (Brown et al 1985). In contrast, several of the SFAs shown in chart 1 exhibit pronounced changes compared to homogeneous solutions when incorporated as "guests" into phospholipid bilayer solutions with strong increases in the monomer fluorescence efficiency and lifetime, particularly in the more rigid low temperature or gel phase (Suddaby et al 1985). For the intrachain SFAs, ${ }_{4} \mathrm{~S}_{6 \mathrm{~A}}$ and ${ }_{6} \mathrm{~S}_{4 \mathrm{~A}}$, the fluorescence efficiencies approach unity and isomerization is virtually eliminated. As indicated above, the SFAs exhibit quite different behavior when incorporated into supported Langmuir-Blodgett multilayers. Typically the SFAs can form pure monolayer films or they can be used in mixtures with saturated fatty acids of similar overall indicated length for the fully extended molecules. Figure 1 compares the solution and LB multilayer assembly fluorescence and absorption for ${ }_{6} \mathrm{~S}_{4 \mathrm{~A}}$. The red shift in fluorescence and concurrent blue shift in absorption can be readily ascribed to the formation of an " $H$ " aggregate which would be predicted for a closely packed array of SFA molecules enforced by film compression (Mooney et al 1984). The types of aggregates that might be anticipated for chromophores such as the SFAs are demonstrated in figure 2 . We originally suspected that " $H$ " aggregate formation should be observed for very concentrated LB films and supported multilayers formed from a single SFA as a consequence of a packing phenomenon, rather than due to any preferential association phenomenon (Mooney et al 1984). Nonetheless we found that aggregate formation is very general for all the SFAs studied 

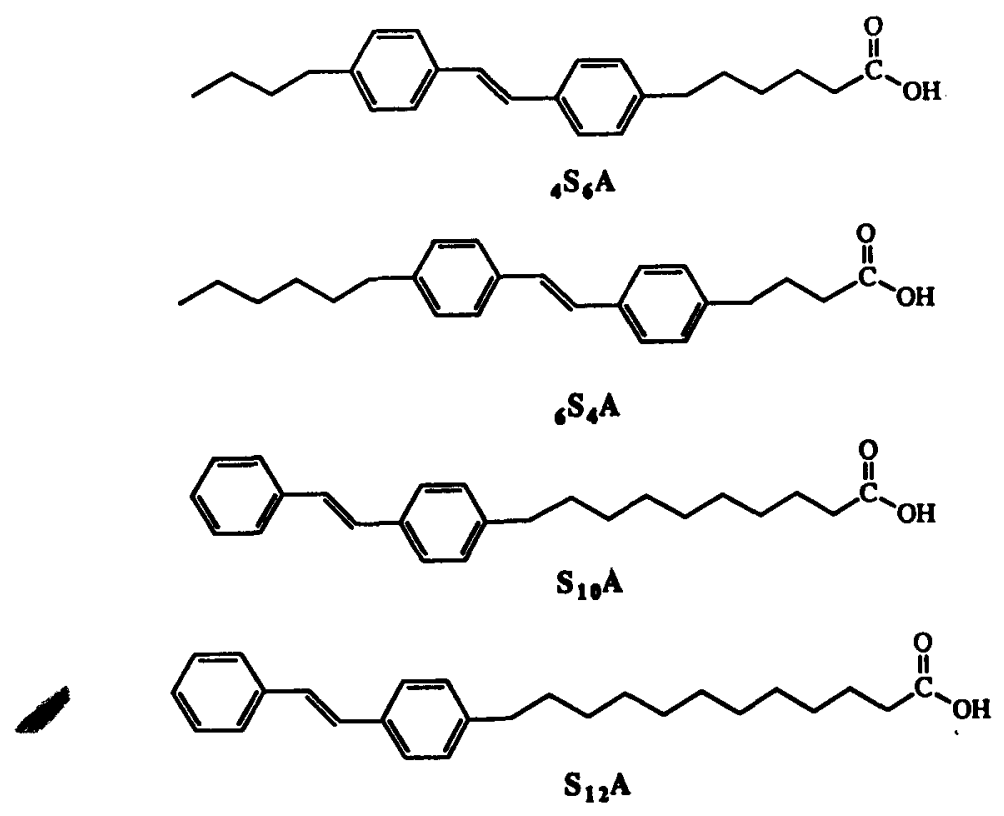<smiles>O=C(O)CCCCCCCCCCCCCCCc1ccc(/C=C/c2ccccc2)cc1</smiles><smiles>CCCCCCCCCCCCCCCCCCCC(=O)O</smiles>

$\mathbf{A A}$

Chart 1.

to date and persists for these compounds to the lowest dilutions (1:20 SFA:fatty acid "host") with little change in the appearance of the aggregate fluorescence or absorption (Spooner et al, unpublished results).

Studies of the aggregate photophysics in assemblies containing potential quenchers which might serve as energy or electron acceptors from the SFAs indicate that the aggregates function as good donors in both cases but are very inefficient in transferring energy between adjacent layers of SFA (Mooney and Whitten 1986). For example, in an earlier study with layers of ${ }_{6} \mathrm{~S}_{4 \mathrm{~A}}$, it was found that while energy transfer (halfquenching distance of $70 \AA$ ) occurs even when the SFA and cyanine quencher are separated by more than one layer, there is no enhancement of the quenching when multilayers of the SFA are placed in contact with a single layer of the cyanine (Mooney and Whitten 1986). This indicates quite clearly that the SFA aggregates are very inefficient at "relaying" energy by degenerate transfer of singlet excitation across adjacent layers and result in no "antenna effect" such as might be anticipated were degenerate energy transfer to occur with reasonable efficiency. This observation is probably reasonable in view of the low oscillator strength of the low energy (long wavelength) transition associated with the $\mathrm{H}$ aggregate and the large "Stokes shift" between fluorescence and absorption. 


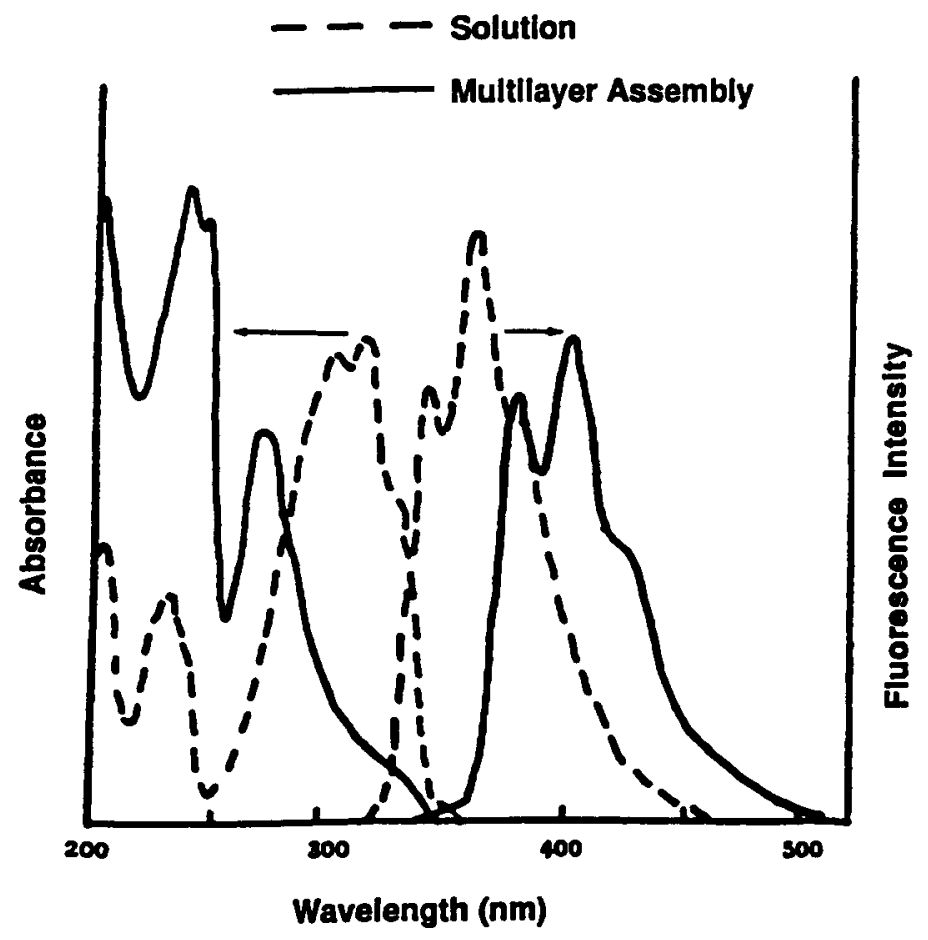

Figure 1. Comparison of solution and multilayer assembly spectra of ${ }_{4} S_{6 \mathrm{~A}}$.
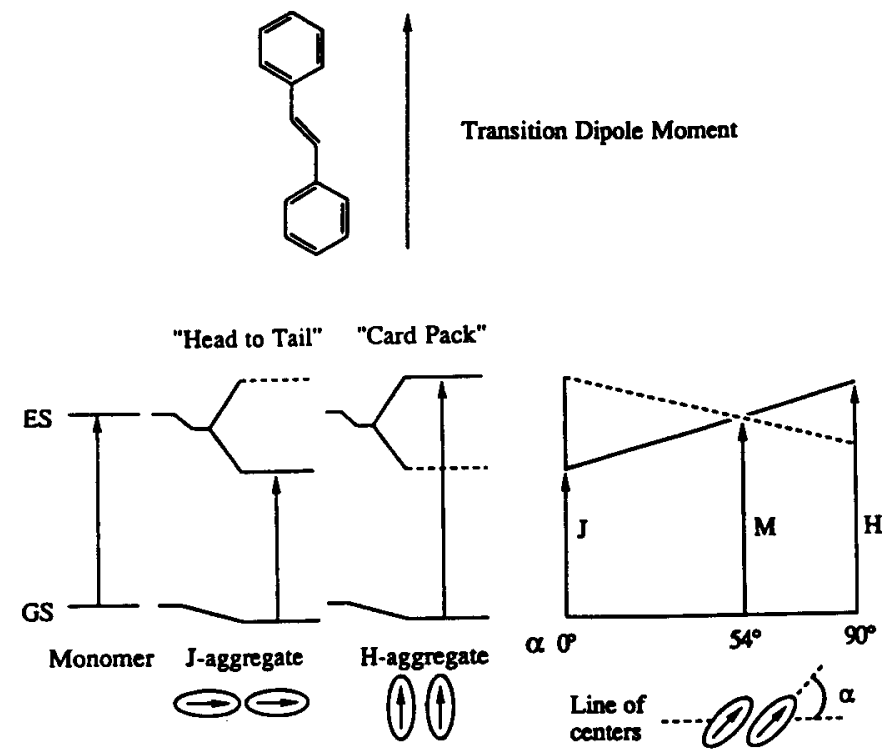

Figure 2. Geometrical arrangement of chromophores leading to the formation of $\mathbf{H}$ and $\mathrm{J}$ aggregates. 


\subsection{Experiments with mixtures of stilbene fatty acids}

The diagram shown in figure 2 suggests that different SFAs might associate to form aggregates having different photophysical properties. Most notable is the prospect that two or more SFAs in an array leading to a large "offset" of the stilbene chromophore might result in an aggregate having " $\mathrm{J}$ " character which could lead to a high oscillator strength and fast fluorescence from the low energy transition with a resultant highly efficient degenerate energy transfer or "antenna effect". Towards this end, we have examined Langmuir-Blodgett assemblies formed from mixtures of three or five SFAs with a saturated fatty acid host. In one case we used a 1:1:1:1:1:5 mixture of ${ }_{4} \mathrm{~S}_{6 \mathrm{~A}},{ }_{6} \mathrm{~S}_{4 \mathrm{~A}}, \mathrm{~S}_{10 \mathrm{~A}}, \mathrm{~S}_{12 \mathrm{~A}}, \mathrm{~S}_{16 \mathrm{~A}}$ and arachidic acid; more extensive investigations have been carried out with a 1:1:1:3 mixture of ${ }_{4} S_{6 A},{ }_{6} S_{4 A}, S_{12 A}$ and arachidic acid. In both cases, relatively similar behavior has been observed (Spooner and Whitten 1991). The absorption spectrum is very similar to that shown for a single SFA in figure 1 and the fluorescence is rather similar (figure 3) and dominated by a relatively long-lived component suggesting a forbidden lowest energy transition similar to the " $\mathrm{H}$ " aggregate observed with a single stilbene. However, a number of studies with asscmblies of different compositions have shown that the SFA mixture gives very different photophysical behavior compared to assemblies of similar composition but with only a single SFA. Fluorescence from a single layer or bilayer of the SFA mixture is readily quenched when a quencher (electron acceptor such as a surfactant viologen or cobalt complex) is incorporated into the same or adjacent layer with hydrophilic-hydrophilic contact. Moreover, quenching by a single layer of quencher can be effective for assemblies containing several sequentially deposited layers as illustrated in figure 4. Thus, for the SFA mixture we observe results consistent with the effective migration or delocalization of excitation over several multilayers but overall spectroscopic characteristics associated with the " $\mathrm{H}$ " aggregate. This raises several questions which we have attempted to address in recent investigations.

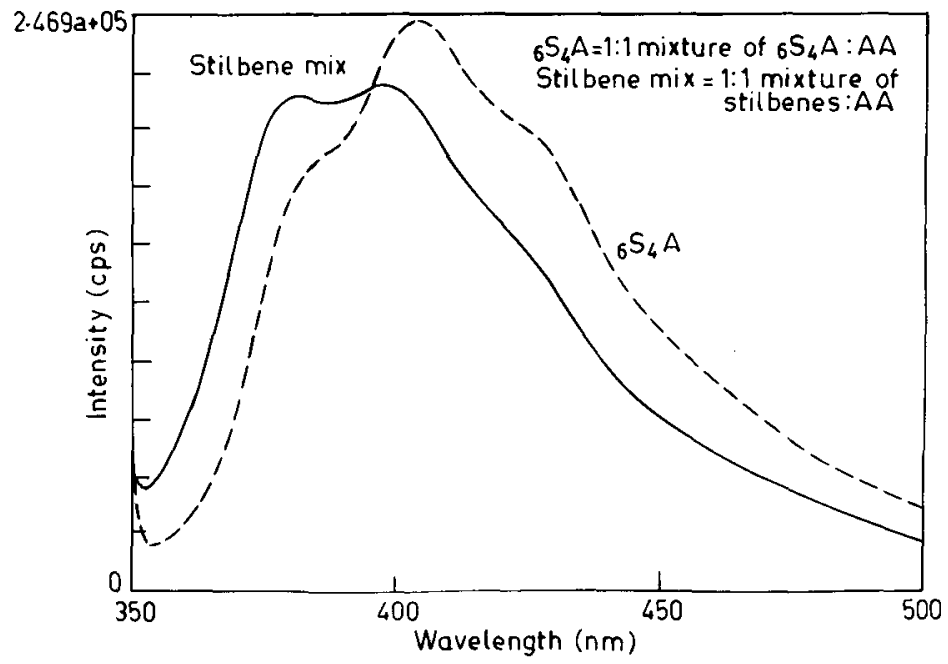

Figure 3. Fluorescence quenching of 5 layers of the stilbene mixtures vs 5 layers of a single type of stilbene. 


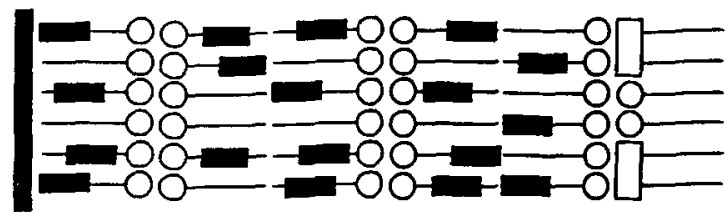

(a)

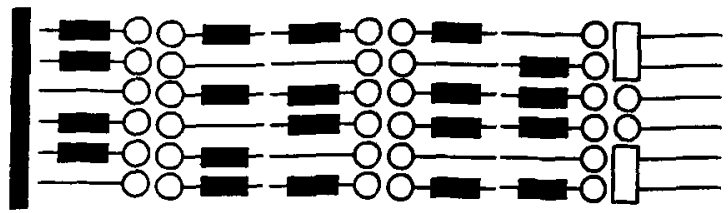

(b)

Figure 4. Fluorescence spectra of ${ }_{6} \mathrm{~S}_{4 \mathrm{~A}}$ and the stilbene mixtures - as compared to a slide containing five layers of the stilbene mixture, the addition of a layer of $\mathrm{V}_{218}$ (as shown above) results in $41 \%(\mathrm{a})$ and $5 \%$ (b) reduction in the fluorescence intensity.

\subsection{Questions concerning the " $H$ " aggregate}

The finding that mixtures containing several SFAs give absorption spectra similar to those for a single SFA or SFA-arachidate mixture and dominated by the relatively sharp "blue-shifted" transition near $270 \mathrm{~nm}$ suggests that the " $H$ " aggregate may represent a selective association of the stilbene chromophore and not simply an association "forced" by packing of the amphiphiles as the films are compressed. Although the SFA mixture films exhibit limiting areas/molecules and high compressibility similar to those of either arachidate or single SFA-arachidate mixtures, the "lift off" (initial rise in surface pressure as the film is compressed) occurs for the mixed films at somewhat larger areas/molecules than for those of simpler composition (Spooner et al, unpublished results). This might be taken as evidence that some association of the SFAs occurs before the films are completely compressed. While various theoretical models of the " $H$ " aggregate suggest a range of sizes from fairly small limiting numbers/aggregate to relatively large numbers of monomers, the persistence of similar aggregate spectra and photophysical properties over a wide dilution range for a single SFA-arachidate mixture suggests that the limiting aggregate probably consists of a relatively small number of monomer units. We shall address the important question of limiting size of the aggregate a little later.

One of the interesting features of the " $H$ " aggregate formed from the SFAs is that it is a rather general process for a series of amphiphiles containing conjugated systems similar to the stilbene chromophore (Spooner et al, unpublished results). Moreover, the absorption spectra of a series of aggregates from different amphiphiles are remarkably similar even in cases where significant substitution occurs that results in rather large differences in the solution monomer spectra. Chart 2 shows the structures of several surfactants we have found exhibiting similar behavior with regard to aggregate formation either in pure films and supported layers or in mixtures with saturated fatty acids of corresponding chain length. Figure 5 compares absorption spectra of several of these aggregates in quartz-supported Langmuir-Blodgett assemblies. While the aggregate absorption spectra are all quite similar, the aggregate fluorescence differs substantially, depending upon the individual chromophores and these differences 


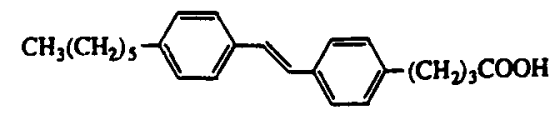

${ }_{6} S_{4 A}$

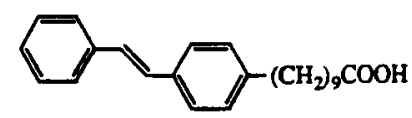

$S_{104}$<smiles>CCCCc1ccc(C=Cc2ccc(C(=O)O)cc2)cc1</smiles>

s $S_{1 A}$

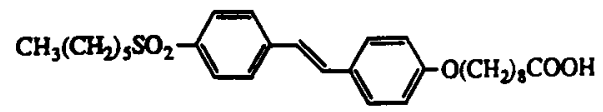<smiles>CCCOc1ccc(C=Cc2ccc(C(=O)O)cc2)cc1</smiles>

$.0 S_{1 \mathrm{~A}}$

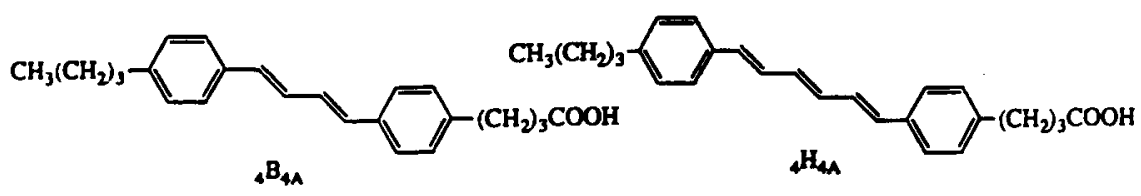

Chart 2.

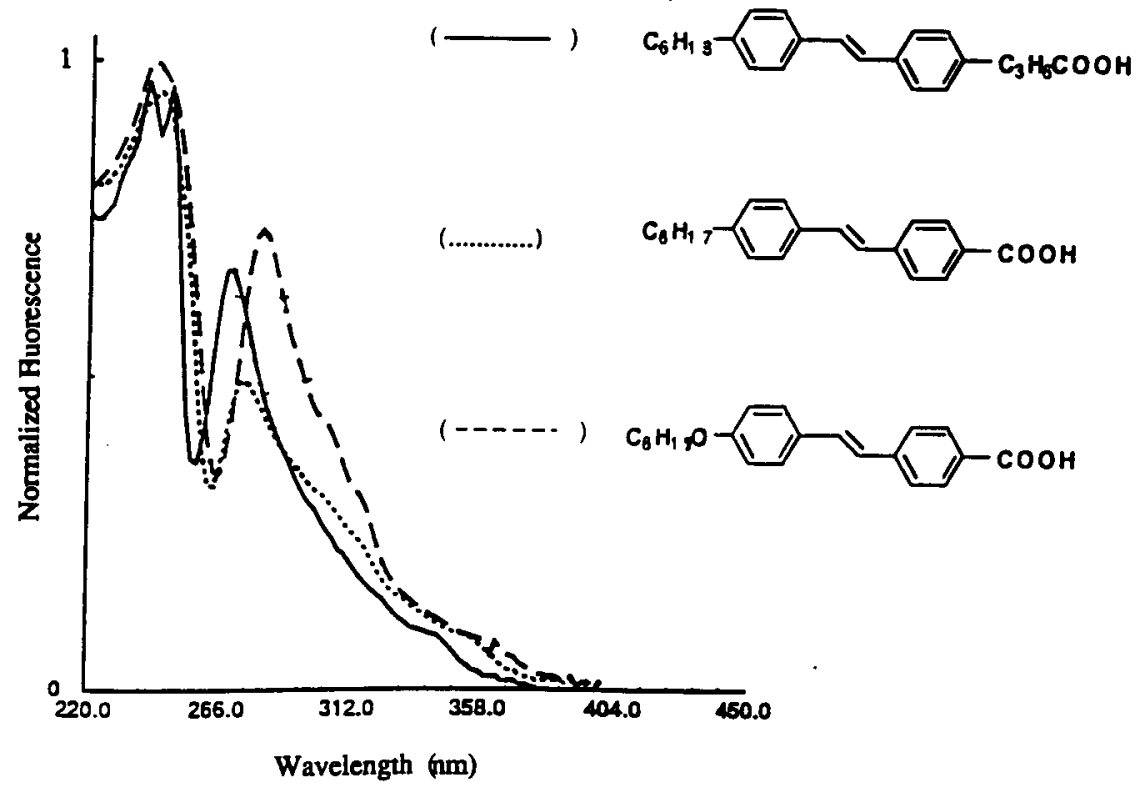

Figure 5. Multilayer absorption of 1:1 mixtures of substituted surfactant stilbenes and arachidic acid. 


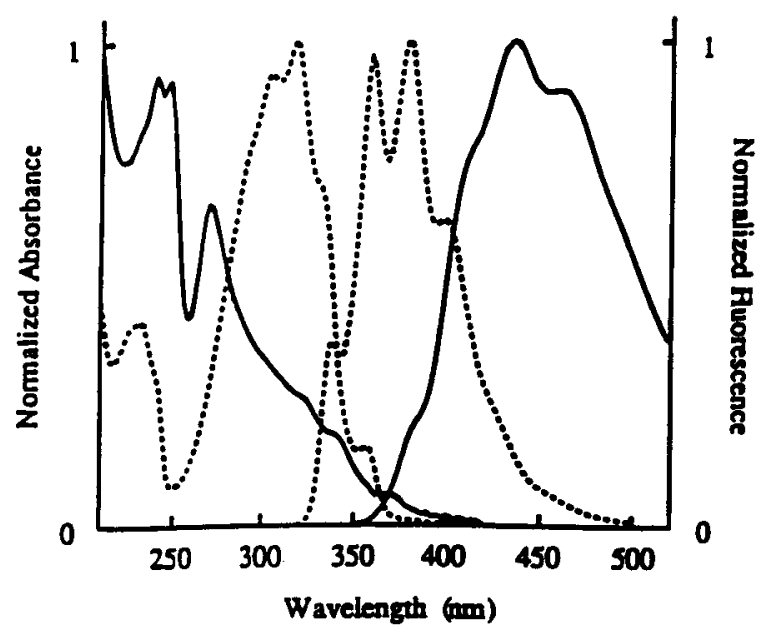

Figure 6. Comparison of solution and multilayer assembly spectra of ${ }_{4} B_{4 A}$.

have not yet been systematically studied. Interestingly, the strength of the aggregate seems to be quite variable and generally decreases with extension of the $\alpha$, $\omega$-diphenylpolyene chain. Thus ${ }_{4} \mathrm{H}_{4 \mathrm{~A}}$ and ${ }_{4} \mathrm{~B}_{4 \mathrm{~A}}$ can be rather readily diluted from "aggregate" to monomer by dilution with arachidate (see figure 6 for ${ }_{4} \mathbf{B}_{4 A}$ ) in contrast to the SFAs. Furthermore, addition of a small amount of functionalized surfactant with a lower energy fluorescence than that from the SFA aggregates results in a fluorescence spectrum dominated by the "guest" indicating that energy transfer within a single LB film layer is very efficient.

While the presence of the "pure" " $\mathrm{H}$ " aggregate is easy to assess in the many cases discussed thus far, either with a single conjugated surfactant or a mixture, the question of different or "mixed" aggregates remains somewhat unresolved. It is tempting to associate the small differences in fluorescence spectra for the SFA mixture shown in figure 3 with a small amount of mixed aggregate (fluorescence occurs at wavelengths longer than pure monomer fluorescence in solution but at wavelengths shorter than those associated with the " $H$ " aggregate). The presence of such a species could also account for the efficient "delocalization" of excitation discussed above in connection with the SFA mixture in LB multilayers. Spectral subtraction of the single SFA aggregate from the mixture provides what might be the spectrum of a "mixed aggregate" (figure 7).

\subsection{Stilbene fatty acid phospholipids}

A different approach, which we have recently initiated, to the study of aggregate formation with the SFA derivatives involves the synthesis and study of stilbene fatty acid esters of phosphatidyl choline (Furman et al, unpublished results). Synthesis of bis-SFA-phosphatidyl choline derivatives is reasonably straightforward; selective enzymatic hydrolysis to the mono-SFA-phosphatidyl choline and esterification with a second SFA afford phosphatidyl choline derivatives containing two different SFAs (Furman et al, unpublished results). Chart 3 shows the structures of the three SFAphosphatidyl choline derivatives that we have initially studied. To date, we have 


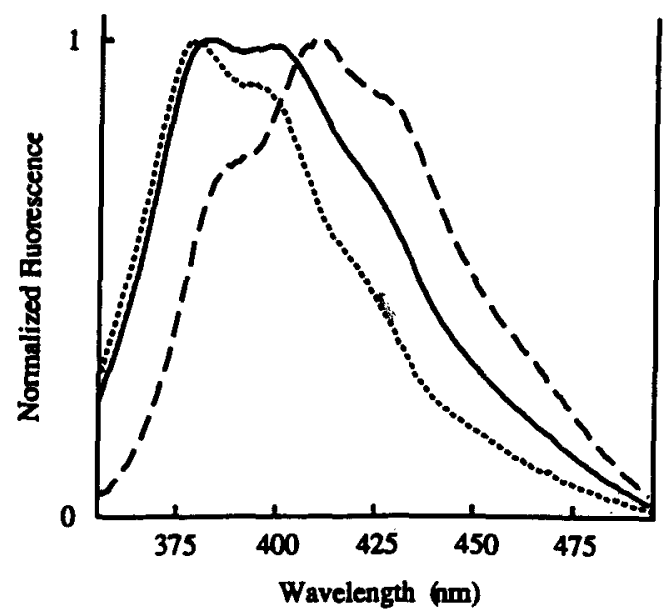

Figure 7. Normalized fluorescence spectra of four component SFA mixture $(1: 1: 1: 7)(-)$ ${ }_{6} \mathrm{~S}_{4 \mathrm{~A}}$ :AA (3:7) (---), and " $\mathrm{H}$ " dimer spectrum resulting from the subtraction of ${ }_{6} \mathrm{~S}_{4 \mathrm{~A}}$ : and AA from SFA mixture ( $\cdots)$.

SFA Phospholipids

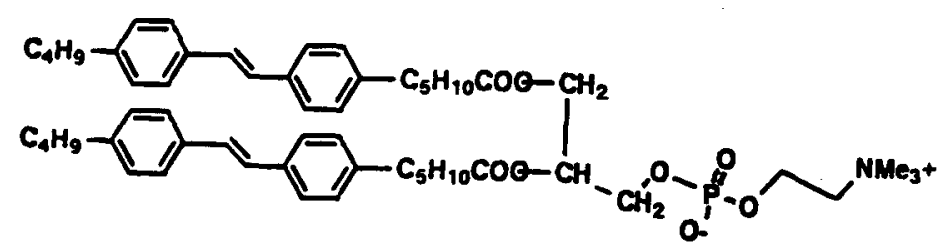

Bis ${ }_{4} S_{6 E} P C$

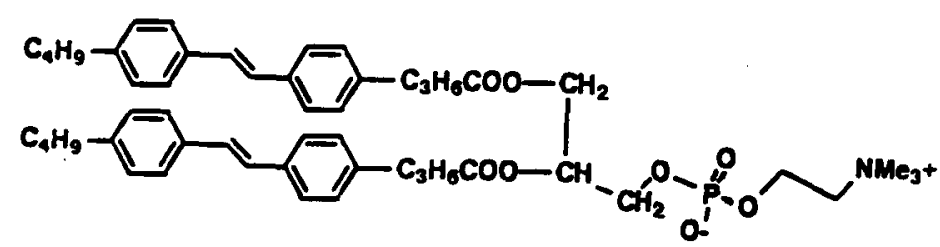

Bis ${ }_{4} S_{4 E} P C$

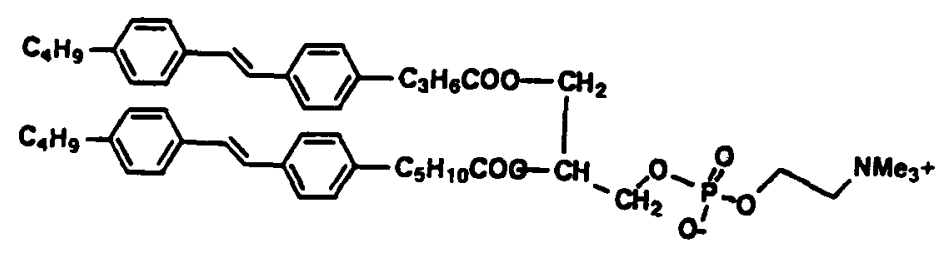

${ }_{4} S_{4-4} S_{6 E}-\mathrm{PC}$

Chart 3. 


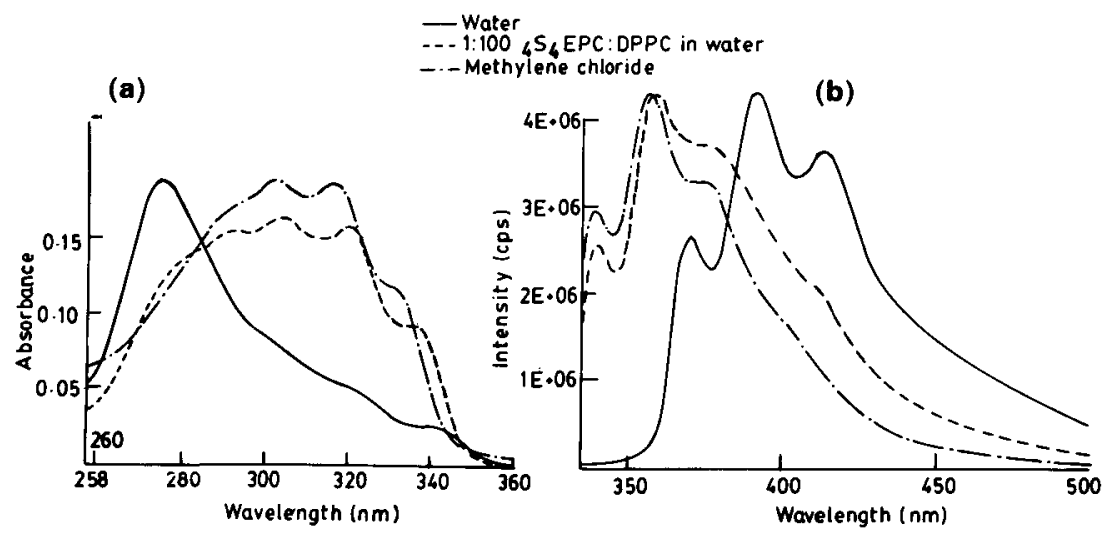

Figure 8. Absorbance (a) and fluorescence spectra (b) for bis ${ }_{4} \mathrm{~S}_{4 \mathrm{E}} \mathrm{PC}$ in water (-),

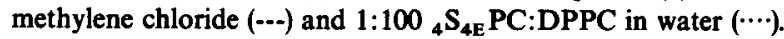

examined the behavior of these three SFA-PC derivatives in dilute organic solvents, in water and in water-containing excess of dipalmitoyl phophatidyl choline (DPPC). We have also examined the behavior of the SFC-PC's in aqueous and aqueous methanol solutions with $\gamma$-cyclodextrin. All three SFC-PC's show essentially identical behavior in dilute methylene chloride solution. The absorption and fluorescence spectra are nearly identical to those of the monomeric stilbene fatty acid (e.g. $\left.{ }_{6} S_{4 A}\right)$ in the same solvent; the fluorescence quantum yield and lifetime are also similar for the SFC-PC's in methylene chloride to that of the SFA monomer. This clearly indicates that in a non-polar or moderately polar aprotic solvent there is little tendency for the transstilbene chromophores of the SFC-PC's to associate with one another, even though the "local concentration" is extremely high. The three SFC-PC's all show rather similar behavior on dispersal in pure water; bot'l the absorption and fluorescence spectra are clearly those of the " $\mathrm{H}$ " aggregate and nearly identical to those obtained in LB assemblies of the SFA's. Some preliminary studies indicate that the organized assemblies formed from pure SFA-PC - presumably bilayer vesicles or multilamellar vesicles - are much larger than those formed by similar treatment of DPPC. The spectral behavior of the SFA-PC's in dispersions 1:100 with DPPC in water is slightly different for each PC but in general different from either monomer or " $\mathrm{H}$ " aggregate (figure 8). The absorption spectra show rather broad bands extending from the range of the " $H$ " aggregate on the blue to slightly beyond the monomer to the red. The fluorescence is predominantly monomer but has at least some contribution from a longer wavelength component.

More illuminating results are obtained from studies of the SFA-PCs in aqueous methanol solutions containing $\gamma$-cyclodextrin (Furman et al, unpublished results). Although pure aqueous solutions give only an " $\mathrm{H}$ " aggregate spectrum, addition of methanol results in conversion to a monomer absorption and fluorescence, indicating that the bilayer aggregates are being dispersed and solubilized into dissolved monomer. However addition of $\gamma$-cyclodextrin results in a change in both absorption and fluorescence as shown in figure 9. The changes are most pronounced for the bis $\left({ }_{4} \mathrm{~S}_{6 \mathrm{E}}\right)$ $P C$ for which both the absorption and fluorescence are clearly that of the " $H$ " aggregate. For bis $\left({ }_{6} \mathrm{~S}_{4 \mathrm{E}}\right)$ PC and $\left({ }_{4} \mathrm{~S}_{6 \mathrm{E}},{ }_{4} \mathrm{~S}_{4 \mathrm{E}}\right) \mathrm{PC}$, the spectra resemble those of the same PC's in diluted aqueous solutions with DPPC as the "host." The results with the bis $\left({ }_{4} \mathrm{~S}_{6 \mathrm{E}}\right)$ 
(a)

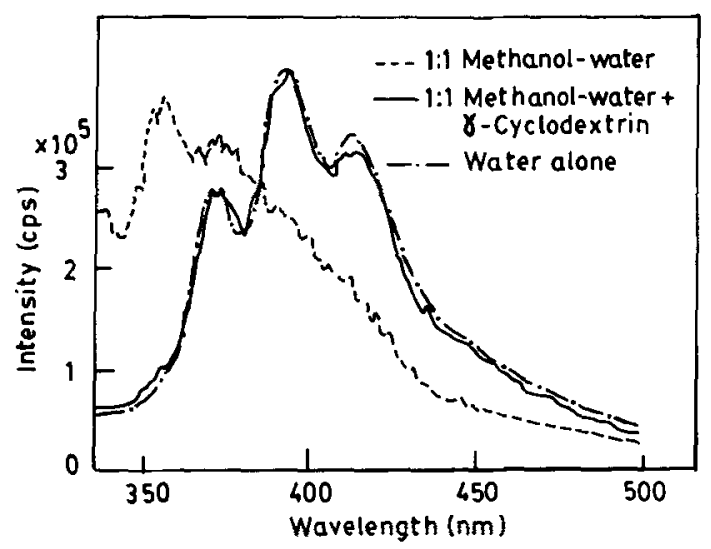

(b)
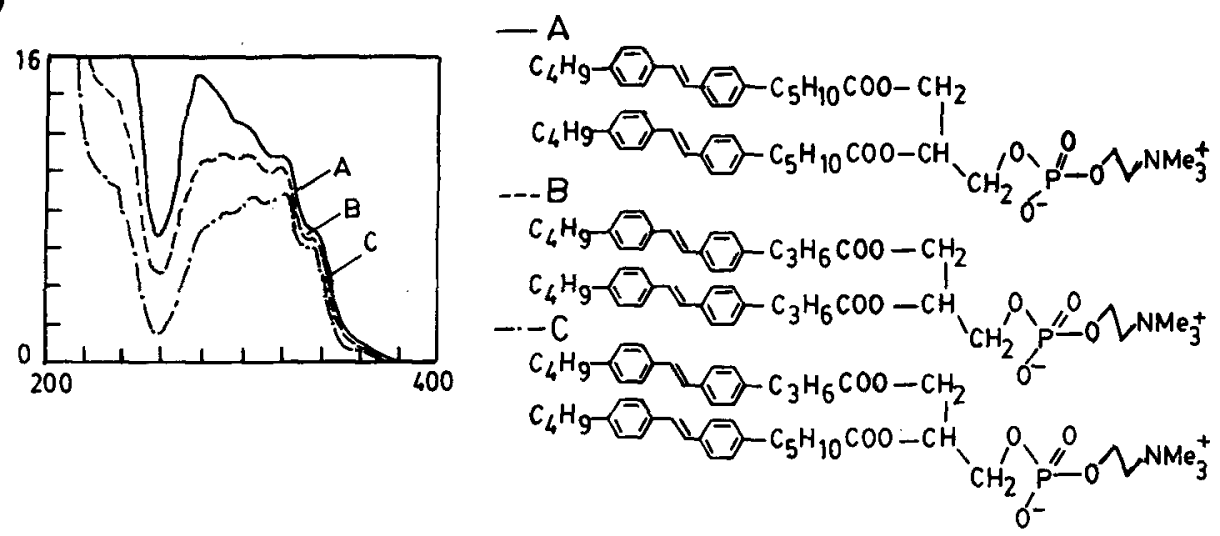

Figure 9. (a) Fluorescence spectra of bis ${ }_{4} S_{6 \mathrm{E}} \mathrm{PC}$ in water, 1:1 methanol-water and 1:1 methanol-water $+y$-cyclodextrin. (b) Absorbance spectra of ${ }_{4} \mathrm{~S}_{6} \mathrm{EPC},{ }_{4} \mathrm{~S}_{4 \mathrm{E}} \mathrm{PC}$ and ${ }_{4} \mathrm{~S}_{6 \mathrm{E}}$, ${ }_{4} \mathrm{~S}_{4 \mathrm{E}} \mathrm{PC}$ diluted 1:100 with DPPC in water.

PC suggest that the minimum " $\mathrm{H}$ " aggregate is a dimer (since models suggest it is impossible to force more than two trans-stilbene units into the cyclodextrin cavity). The difference between this PC and the other two examined thus far is ascribed to the distance of the stilbene chromophore from the phosphatidyl choline head group and glycerol backbone. For the two PC's containing shorter chains it appears reasonable that there is insufficient flexibility for the two stilbenes to form the "ideal" " $\mathrm{H}$ " dimer and so forced association leads to an aggregate having different spectroscopic properties. In the case of the longer chain separation, ideal " $\mathrm{H}$ " aggregate geometry can be attained and the spectroscopic properties are almost identical to those in the extended bilayer or multilayer assemblies.

\section{Conclusions}

While we are still examining many aspects of the controlled formation of aggregates in LB assemblies and phospholipid bilayers, the results obtained thus far suggest that the limiting spectral shifts for the aggregate can be attained with an "H" dimer and that this species does indeed represent an energy minimum. The association to form 
the "H" aggregate or dimer is evidently closely related to apolar association of other aromatic chromophores in aqueous media (Dewey et al 1978; Diederich 1990; Smithrud et al 1990) and may exert important control over the type of assembly formed by these aromatic-functionalized amphiphiles. While the " $H$ " aggregate clearly represents an energy minimum or at least a favored aggregate, our studies with the phospholipids, and to some extent the LB assemblies, suggest that packing forces can in some cases lead to the formation of different aggregates having quite different photophysical properties which may be of interest in tuning the behavior of extended arrays of these chromophores. While these arrays of organic chromophores may be thought of as "supramolecular" species having a commonality with inorganic quantum species such as clusters or semiconductor colloids (Lehn 1988; Kortan et al 1990), the stereochemical complexity of the organic chromophores makes their behavior potentially much more complex and possibly quite versatile.

\section{Acknowledgements}

We are grateful to the US National Science Foundation (grant number CHE 9211586) for support of this research.

\section{References}

Allen M T and Whitten D G 1989 Chem. Rev. 891691

Brown P E, Mizutani T, Russell J C, Suddaby B R and Whitten D G 1985 ACS Symp. Ser. 278171

Dewey T G, Wilson P S and Turner D H $1978 \mathrm{~J}$. Am. Chem. Soc. 1004550

Diederich F 1990 J. Chem. Edu. 67813

Furman I, Geiger H C, Richard W and Whitten D G (unpublished results)

Geiger M W and Turro N 1977 J. Photochem. Photobiol. 26221

Hammond G S et al 1964 J. Am. Chem. Soc. 863197

Itoh T and Kohler B E 1987 J. Phys. Chem. 911760

Kortan A R, Hull R, Opila R L, Bawendi M G, Steigerwald M L, Carroll P J and Brus L E $1990 \mathrm{~J}$. Am. Chem. Soc. 1121327

Lehn J-M 1988 Angew. Chem., Int. Ed. Engl. 2790

Mizutani T and Whitten D G $1985 \mathrm{~J}$. Am. Chem. Soc. 1073621

Mooney W F III 1983 Energy and electron transfer in multilayer assemblies of surfactant stilbene derivatives $\mathrm{Ph} \mathrm{D}$ dissertation, University of North Carolina, Chapel Hill

Mooney W F III, Brown P E, Russell J C, Costa S B, Pedersen L G and Whitten D G 1984 J. Am. Chem. Soc. 1065659

Mooney W F and Whitten D G 1986 J. Am. Chem. Soc. 1085712

Russell J C, Costa S B, Seiders R P and Whitten D G $1980 \mathrm{~J}$. Am. Chem. Soc. 1025678

Russell J C, Whitten D G and Braun A M 1981 J. Am. Chem. Soc. 1033219

Saltiel J 1992 J. Photochem. Photobiol. 6529

Saltiel J, Chang D W L, Megarity E D, Rousseau A D, Shannon P T, Thomas B and Uriarte A K 1975 Pure Appl. Chem. 41559

Saltiel J and Charlton J L 1980 In Rearrangements in ground and excited states (ed.) P deMayo (New York: Academic Press)

Smithrud D B, Sanford E M, Chao I, Ferguson S B, Carcanague D R, Evanseck J D, Houk K N and Diederich F 1990 Pure Appl. Chem. 122227

Spooner S P, Furman I, Geiger H C and Whitten D G (unpublished results)

Spooner S P and Whitten D G 1991 Proc. Soc. Photo-Opt. Instrum. Eng. 821436

Suddaby B R, Brown P E, Russell J C and Whitten D G 1985 J. Am. Chem. Soc. 1075609

Takagi K, Suddaby B R, Vadas S L, Backer C A and Whitten D G 1986 J. Am. Chem. Soc. 1087865 